\title{
Telepathological evaluation of paediatric histological specimens in support of a hospital in Tanzania
}

\author{
Hans-Ullrich Voelker ${ }^{1}$, Laura Poetzl ${ }^{1}$, Annette Strehl ${ }^{1}$, Hans-Konrad Mueller-Hermelink ${ }^{2}$, \\ Ansgar Stuefe ${ }^{3}$, Gerhard Stauch ${ }^{4}$
}

1. Pathology, Leopoldina Hospital, Gustav-Adolf-Str 8, D-97422 Schweinfurt.

2. Institute of Pathology, University of Wuerzburg, Josef-Schneider-Str. 2, D-97080 Wuerzburg.

3. Abbey Muensterschwarzach, Schweinfurter Str 40, D-97359 Münsterschwarzach.

4. Parkstraße 62, D-26605 Aurich.

\begin{abstract}
Background/Objective: In a project of telepathology (TP) between German pathologists and a hospital in Tanzania, trained technical assistants have uploaded digital histological images onto the internet-based platform ipath. The diagnoses from 486 paediatric specimens were analysed.

Methods: The investigation included diagnoses, either primarily done via TP or secondarily after a further workup of the paraffin-embedded tissue, which was sent to Germany for cases which could not be solved via TP. In the latter, the initial TP-diagnoses were compared with the results after re-evaluation.

Results: The median age was 11 years. The cohort comprised 390 benign diseases (80.2\%) and 96 malignant diseases $(19.8 \%)$. For benign diseases, the most frequent anatomic sites were lymph nodes, skin, and soft tissue, breast, and head\&neck. Frequent diagnoses were non-specific inflammations and benign tumors. In malignant diseases, the most sites were lymph nodes, skin, soft tissue, head\&neck, and ovary and the most frequent diseases sarcomas and lymphomas. The paraffin embedded tissue of 179 cases (36.3\%) was shipped to Germany. With the concordance analysis, we could discover the mandatory necessity for the possibility of second opinion in difficult cases.

Conclusion: An exclusively TP-support cannot meet all requirements of modern medical diagnostics. The education of local pathologists is imperative.

Keywords: Telepathology, low income country, ipath, paediatric.

DOI: https://dx.doi.org/10.4314/ahs.v20i3.37

Cite as: Voelker H-U, Poetzl L, Strehl A, Mueller-Hermelink H-K, Stuefe A, Stauch G. Telepathological evaluation of paediatric histological specimens in support of a hospital in Tanzania. Afri Health Sci. 2020;20(3): 1313-1321. https:// dx.doi. org/10.4314/abs.v20i3.37
\end{abstract}

\section{Background}

The St. Josef's Mission Hospital in Peramiho (SouthWest of Tanzania) is maintained by missionary Benedictines (http://www.peramiho.org/en/health/hospital.html, accessed April 20 ${ }^{\text {th }}$ 2020). It has around 320 beds, basic medical wards (e.g. surgery, gynecology, pediatrics, tuberculosis-ward) and cares for more than 100.000 out- and inpatients per year. Medical services in many low income countries need reliable histopatho-

\section{Corresponding author: \\ Hans-Ullrich Voelker, \\ Pathology Leopoldina Hospital \\ Gustav-Adolf-Str. 8 D-97422 Schweinfurt \\ Phone +49 9721-720-3530 \\ Fax +49 9721-720-2996 \\ Email: hvoelker@leopoldina.de}

logical reports for effective treatment planning, especially against the background of limited resources ${ }^{1}$.

In 2001, Oberholzer and Brauchli presented the internet-based platform ipath (https://www.ipath-network. $\mathrm{com} /$ tanzania/, April $\left.20^{\text {th }} 2020\right)$ for the purpose of static telepathology (TP). The system was developed at the University of Basel, Switzerland, for communication between generalists and consultant specialists in pathology on the basis of digital images of histological cases (in this method, in contrast to dynamic TP, there was no utilization of real-time images). The first project of consultation was initiated on the Island of Samoa ${ }^{2,3}$. In 2007, we started a collaboration with the hospital in Tanzania which cannot employ local pathologists due to financial constraints. The application of TP in cooperation with educated technical assistants and remote consultant pathologists was integrated into the diagnostic process - to our knowledge a new way.

C 2020 Voelker H-U et al. Licensee African Health Sciences. This is an Open Access article distributed under the terms of the Creative commons Attribution License (https://creativecommons.org/licenses/BY/4.0), which permits unrestricted use, distribution, and reproduction in any medium, provided the original work is properly cited. 
The project was based on the evaluation of biopsy specimens for the establishment of a primary histopathological diagnosis without the necessity for evaluation of whole specimens from surgery. In this setting there was no requirement for the assessment of resection margins or tumor stages.

A laboratory was set up with technical facilities for histological examination using hematoxylin-eosin stains (H\&E). There was no possibility for special stains or immunohistochemistry. Local technical assistants were given special training over ten weeks to cover the processing and paraffin-embedding of tissue, the staining of slides and the microscopic evaluation of the finished cases (with the intention of identifying areas of divergent morphology independent of their diagnostic significance). These areas were documented with digital images (*.jpeg) and uploaded onto ipath with a short clinical history and clinical issues. The final diagnoses were communicated by the participating consultant pathologists. For the first 545 cases we could show the diagnostic validity of our method. These cases were investigated primarily via $\mathrm{TP}$, after which the paraffin embedded tissue was sent to the University of Wuerzburg, Germany, for a secondary workup. A complete concordance was found in $70 \%$ and only mild discordances (e.g. divergent grading of cancer or other cause of inflammatory processes) in $14 \%$. In this way a clinically useful diagnosis could be given in $84 \%$, despite limited resources ${ }^{4}$. This result is not consistent with the requirements stipulated for regular histopathological services, but is provisionally acceptable in the given local setting and comparable with results found in the literature ${ }^{5-7}$.

The aim of the study presented here was to analyse diagnostic findings generated from specimens of children and adolescents up to 18 years, extracted from the total of 5230 cases (mostly adults) which were diagnosed via TP over a period of ten years in the ongoing project. The aim of this study was not to re-evaluate clinical consequences arising from the given diagnoses or their implementation in the further treatment of patients, because of the high degree of heterogenicity of the individual patient's situation and the available therapeutical options.

\section{Methods}

The analysis comprises diagnostic findings from specimens of children and adolescents in the course of our TP-project from April 2007 to April 2017. The investigation included all final diagnoses, of which some were made primarily via TP and others secondarily af- ter a further workup of the paraffin-embedded tissue, which was sent to Germany for those cases which could not be solved via TP. All cases were archived in ipath and transformed into a tabulated calculation standard. Computation was done using Microsoft Excel ${ }^{\circledR}$.

All patient data was completely anonymised. No individual patient data was released (specifically name, date of birth, or reference number of hospital). Instead all cases were assigned consecutive numbers according to their order of appearance by the local technicians, who then merged the incoming case information with their patient information and forwarded this to the local physicians.

The anatomic locations from which the specimens were taken were divided into 17 groups. To enable a detailed analysis the diagnoses were grouped into ten benign and eight malignant main categories (table 1).

For the majority of cases the gender and age of the patients was known. From this the median, average, minimum, and maximum ages was computed. Additionally the median, average, minimum, and maximum elapsed time between the upload of the case and the finished diagnosis was calculated as well as the number of uploaded histological images. The disclosed clinical history of the concerned patients and the suspected diagnosis made by the technical assistants in Peramiho were not analysed because of non-standardized formatting for the former and limited pathological experience for the latter.

The five anatomic sites with the highest number of cases were investigated for their two most frequent diagnoses.

In cases which were found to be difficult to diagnose solely via TP the shipment of paraffin embedded tissue to Germany was requested by the consultant pathologists to obtain a second opinion. The reason for request was: poor quality of specimen or images, diagnoses which need special stains or immunohistochemistry for confirmation, or rare diagnoses. After their arrival these specimens were processed according to routine diagnostic standards for histopathological examination using H\&E-stained slides, special stains, and immunohistochemistry. As a result, an independent second opinion given by German university-standard could be obtained. It was not the intention of this investigation to compare the primary H\&E-based telepathological diagnosis with a second H\&E-based diagnosis by German pathologists using slides created in Germany but rather to test how well the TP diagnosis correspond with a 
comprehensive diagnosis made using a full spectrum of methods. Subsequently, the diagnoses generated during this secondary appraisal were compared with the main initial diagnoses on TP, with the final diagnoses of the German university being accepted as correct.

In cases where a secondary workup was necessary, the initial TP-diagnoses were compared with the final results with evaluation of the following criteria: 1-completely identical diagnosis in TP and in the second opinion; 2-mild discordance, i.e. correct diagnosis, but e.g. divergent grading of cancer or cause of inflammatory processes; 3-correct distinction between benign and malignant process but differing disease entity (e.g. neurofibroma vs. dermatofibroma or undifferentiated carcinoma vs. sarcoma), 4-false benign in TP; 5 -false malignant in TP; 6-no primary diagnosis possible via TP (e.g. due to difficulties in the primary assessment or the obligation of immunohistochemical stains). The sum total of all completely identical diagnoses and diagnoses with mild discordances were categorised as clinically useful diagnoses. Classical concordance analysis (e.g. Goodman's analysis or Chi-square test) was not suitable for the categorization of these different types of comparable results. Therefore, following a statistician's advice, the results are presented in a descriptive manner with absolute and percentage numbers.

\section{Results}

Over a period of ten years a total of 5230 cases were investigated using static TP. 504 of these cases (9.6\%) were sampled from children and adolescents of up to 18 years. Of these cases, 18 were not suitable for diagnostic purposes because of the insufficient quality of the taken specimen, so that in total 486 cases could be given a final diagnosis. The 18 cases with diagnostically insufficient material include cases containing only very sparse tumor cells, a high degree of artificial alteration due to biopsy sampling or tissue considered non-representative for the clinical question (such as bland soft tissue in a case of a rapidly progressing tumor). Therefore, these cases were primarily excluded. 205 patients each were defined as male or female, three as hermaphrodites. In 73 cases the gender was not known.

The median age was 11 years (average 10.3, range 1 month - 18 years). For histopathological analysis, a median of 9 histological pictures (average 9.5, range 1-48) were uploaded to TP. The corresponding diagnostic report was made by the consultant pathologists after a median of 26 hours (average 67.8, range $1-252$ hours). For confirmation of difficult and/or challenging TP-diagnoses or for additional investigations using special stains and immunohistochemical methods, the paraffin embedded tissue of 179 cases (36.3\%) was shipped to Germany. Shipping times varied between three and eight weeks due to the fact that several cases were collected in the local hospital and then shipped together. In 33 cases the paraffin blocks were not sent despite being requested by the consultants. 
Table 1: Overall distribution of cases to anatomic sites and diagnostic groups $(n=486)$

\begin{tabular}{|c|c|c|c|}
\hline Anatomic sites & $\mathrm{n}$ & Diagnostic groups & $\mathbf{n}$ \\
\hline Abdomen & 9 & Benign diseases & \\
\hline Bone & 23 & Benign tumor & 73 \\
\hline Breast & 53 & Fibroadenoma & 36 \\
\hline Colon & 22 & Fibrotic changes & 25 \\
\hline Eye & 13 & Hirshsprung disease & 7 \\
\hline Fluid & 5 & Non-specific inflammation & 103 \\
\hline Head\&Neck & 39 & Non-specific lymphadenitis & 66 \\
\hline Kidney & 8 & Physiological tissue & 12 \\
\hline Lymph node & 116 & $\begin{array}{l}\text { Reactive benign conditions } \\
\text { (e.g. abortus, mastopathia) }\end{array}$ & 39 \\
\hline Ovary & 14 & Schistosomiasis & 4 \\
\hline Skin & 83 & Tuberculosis & 25 \\
\hline Soft tissue & 75 & Malignant diseases & \\
\hline Testis & 14 & Adenocarcinoma & 8 \\
\hline Thyroid & 2 & Kaposi sarcoma & 5 \\
\hline Urinary bladder & 5 & Lymphoma & 31 \\
\hline Uterine cervix & 2 & Other malignant tumors & 8 \\
\hline \multirow[t]{4}{*}{ Uterus } & 3 & Retinoblastoma & 7 \\
\hline & & Sarcoma & 27 \\
\hline & & Sex cord stroma tumor & 5 \\
\hline & & Squamous cell carcinoma & 5 \\
\hline
\end{tabular}

The complete cohort comprised 390 benign diseases $(80.2 \%)$ and 96 malignant diseases $(19.8 \%)$. In the group of benign diseases, the median age of the 159 female, 161 male, 3 hermaphrodites and 67 patients of unknown gender was 11 years (average 10.2, range 1 month - 18 years). Table 2 shows the most frequent anatomic sites with the most frequent groups of diagnoses. Figure 1 illustrates the subtypes of found benign tumors and the subgroups of benign reactive condi- tions. Of the diagnosed infectious diseases, 25 cases were from lymphonodal tuberculosis and four cases showed schistosomiasis. Of these, two were located in the vulva and each one in a lymph node and the appendix vermiformis. From benign diseases, 107 cases were secondarily investigated in Germany (27.4\%). For 15 cases the corresponding paraffin blocks were not shipped despite being requested. The concordance analysis of these cases is given in table 3 . 


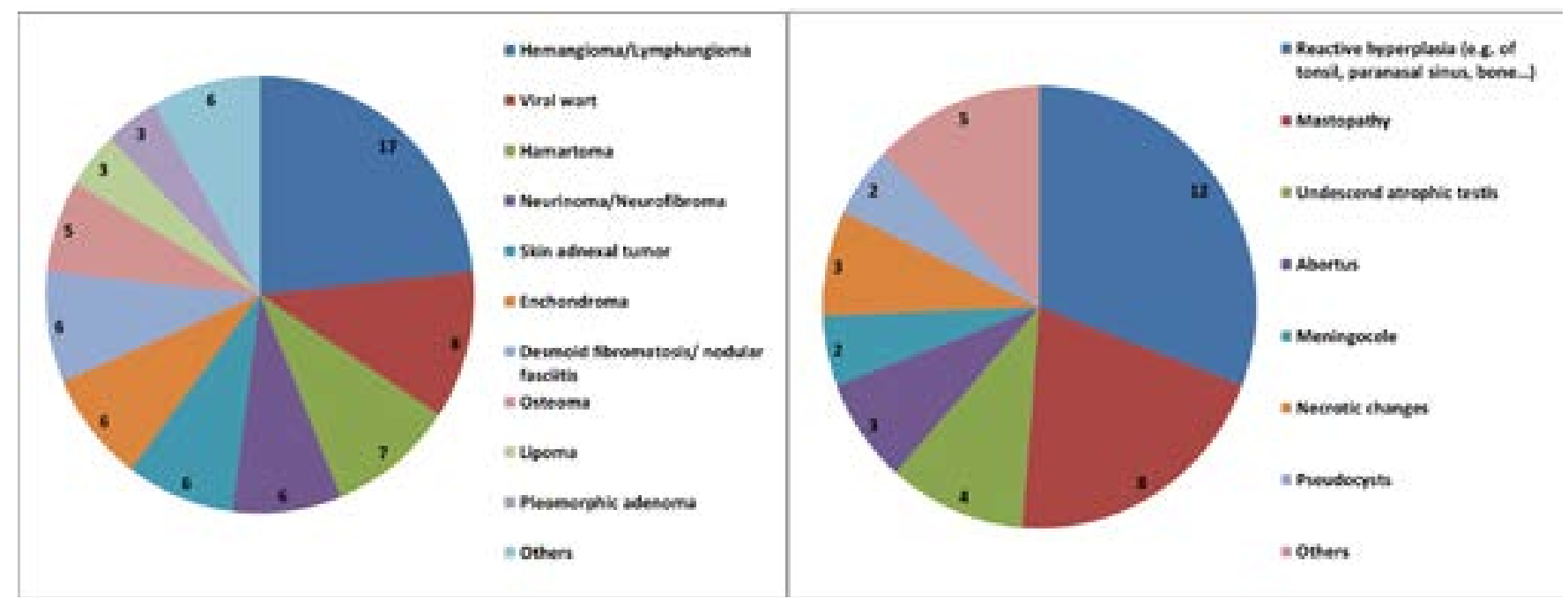

Figure 1: Subtypes of benign tumors (left) and reactive benign conditions (right)

Table 2: The five most frequent anatomic sites and the two most frequent diagnoses of benign and malignant diseases.

\begin{tabular}{|l|l|}
\hline Benign diseases & \\
\hline Lymph node $(n=96)$ & $\begin{array}{l}\text { Non-specific lymphadenitis } n=66 \\
\text { Tuberculosis } n=25\end{array}$ \\
\hline Skin ( $n=73)$ & $\begin{array}{l}\text { Non-specific inflammation } n=32 \\
\text { Benign tumor NOS } n=29\end{array}$ \\
\hline Soft tissue $(n=63)$ & $\begin{array}{l}\text { Non-specific inflammation } n=24 \\
\text { Benign tumor NOS } n=14\end{array}$ \\
\hline Breast ( $n=52)$ & $\begin{array}{l}\text { Fibroadenoma } n=36 \\
\text { Reactive benign conditions } n=8\end{array}$ \\
\hline Head\&Neck $(n=27)$ & $\begin{array}{l}\text { Non-specific inflammation } n=11 \\
\text { Benign tumor NOS } n=10\end{array}$ \\
\hline Malignant diseases & $\begin{array}{l}\text { Lymphoma } n=19 \\
\text { Sarcoma } n=1\end{array}$ \\
\hline Lymph node $(n=20)$ & $\begin{array}{l}\text { Sarcoma } n=10 \\
\text { Lymphoma } n=2\end{array}$ \\
\hline Soft tissue $(n=13)$ & $\begin{array}{l}\text { Adenocarcinoma } n=6 \\
\text { Sarcoma } n=2 \\
\text { Kaposi sarcoma } n=2\end{array}$ \\
\hline Head\&Neck $(n=12)$ & $\begin{array}{l}\text { Squamous cell carcinoma } n=3 \\
\text { Kaposi sarcoma } n=3\end{array}$ \\
\hline Skin $(n=10)$ & $\begin{array}{l}\text { Lymphoma } n=4 \\
\text { Sex cord stroma tumor } n=3\end{array}$ \\
\hline Ovary $(n=10)$ &
\end{tabular}


Table 3: Concordance of diagnoses primarily investigated by telepathology (TP) with a second opinion (SO) in Germany. Diagnostic evaluation: 1-completely identical diagnosis between TP and SO, 2-mild discordances (e.g. in subtype of inflammation or grading of cancer), 3-identical in benign/malignant group, but other entity of disease, 4-Diagnosis of TP was false malignant, 5-diagnosis of TP was false benign, 6- primary diagnosis by TP was not possible.

\begin{tabular}{|c|c|c|c|c|}
\hline & $\begin{array}{l}\text { Sent for } \\
\text { second } \\
\text { opinion }\end{array}$ & $\begin{array}{c}\text { Kind of } \\
\text { concordance }\end{array}$ & $n / \%$ & $\begin{array}{l}\text { Number of cases in } \\
\text { which } \\
\text { immunohistochemistry } \\
\text { (IHC) or special stains } \\
\text { (S) were needed }\end{array}$ \\
\hline \multicolumn{5}{|l|}{$\begin{array}{l}\text { Benign } \\
\text { diseases }\end{array}$} \\
\hline \multirow[t]{6}{*}{390} & 107 & 1 & $50(46.7 \%)$ & S: $14, \mathrm{IHC} 3$ \\
\hline & & 2 & $23(21.4 \%)$ & S: 8, IHC: 3 \\
\hline & & 3 & $22(20.5 \%)$ & S: 11, IHC: 7 \\
\hline & & 4 & $6(5.6 \%)$ & S: 3, IHC: 1 \\
\hline & & 5 & 0 & \\
\hline & & 6 & $6(5.6 \%)$ & $S: 3$ \\
\hline \multicolumn{5}{|l|}{$\begin{array}{l}\text { Malignant } \\
\text { diseases }\end{array}$} \\
\hline \multirow[t]{6}{*}{96} & 72 & 1 & $21(29.1 \%)$ & S: $1, I H C: 17$ \\
\hline & & 2 & $16(22.2 \%)$ & $\mathrm{S}: 1, \mathrm{IHC}: 14$ \\
\hline & & 3 & $18(25.0 \%)$ & IHC: 18 \\
\hline & & 4 & 0 & \\
\hline & & 5 & $11(15.3 \%)$ & S: 2, IHC: 9 \\
\hline & & 6 & $6(8.3 \%)$ & IHC: 3 \\
\hline
\end{tabular}

In the group of malignant diseases, the 46 female, 44 male and the 6 patients of unknown gender showed a median age of 9 years (average 10.0, range 7 months - 18 years). The most frequent anatomic sites and diagnoses are given in table 2 . The subtypes of sarcomas and lymphomas are illustrated in figure 2 . Of the eight reported adenocarcinomas, 6 were found to be primary tumors of salivary glands. Herein we found 3 mucoepidermoid carcinomas, 2 adenocarcinomas ex pleomorphic adenoma and one adenoid-cystic carcinoma. Two further adenocarcinomas were reported as metastases of an unknown primary, in consideration of the immunohistochemical phenotype most likely originating from the gastrointestinal tract. Of the remaining malignant tumors $(n=8)$, three were found to be Wilms tumors of the kidney, one a malignant melanoma, one a neuroendocrine carcinoma G3, one papillary carcinoma of the thyroid, one immature teratoma and one malignant solitary fibrous tumor (formerly hemangiopericytoma). The frequency of necessary secondary opinions was high in this group with 72 cases $(75.0 \%)$, however in 18 cases the block was not shipped despite being requested. The analysis of concordance is given in table 3 . 

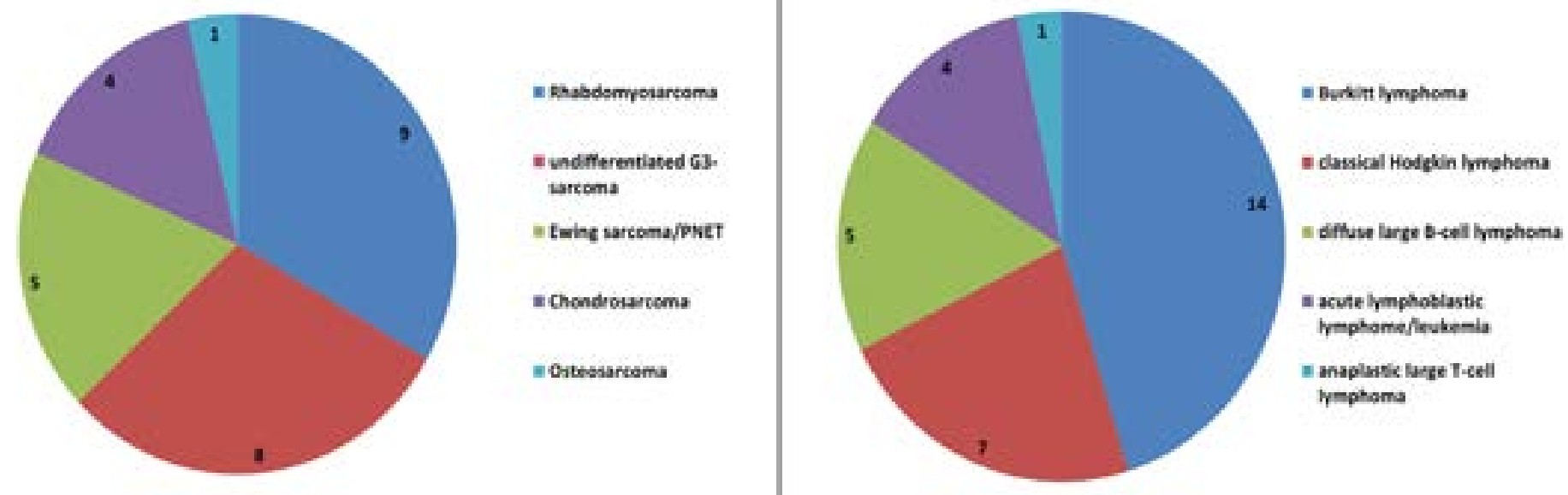

Figure 2: Subtypes of sarcomas (left) and lymphomas (right)

\section{Discussion}

An efficient approach towards histopathological diagnostics is a key element in the advancement of health care systems. The global access to the internet leads to increasing possibilities in telemedicine ${ }^{8}$. A rapid histological investigation can open up the possibility for better therapeutic decisions. Our TP-project between a hospital in Tanzania and consultant pathologists at a German university is well established since ten years. We could establish a good level of diagnostic accuracy in the initial stages of the project, adapted to meet the specific requirements of the currently available medical care $^{4}$.

The processing of cases could usually be concluded in a matter of days. In some cases delays occurred, usually at times when the local laboratory was irregularly staffed or when the quantity of incoming samples was low and the technicians collected samples to process at a later stage. Measured in terms of an average length of stay in the missionary hospital of 4.5 days, these diagnostic reports are nonetheless directly useful. In addition patients with complex diseases usually have a longer than average stay. Diagnostic reports which are received subsequently are often not easily integrated into the treatment plan.

From a collective of 5230 patients we extracted 486 evaluable cases from children and adolescents. 18 samples could not be processed further due to the insufficient quality of the material. The literature does not give information concerning the default rate of tissue samples during histological processing; however, experiences obtained during the establishment of biobanks in similar regions display also sometimes limitations of sample quality ${ }^{9}$. In the whole processing chain logistical and climatic particularities of rural regions in Africa have a detrimental effect on the samples, which is not easily to avoid. From the experiences made during the secondary workup in Germany we also learned thaa further workup for research purposes was not always possible. In an undocumented number of cases additional molecular studies were initiated (for instance PCR for tuberculosis, translocation analysis for lymphoma or sarcoma) but did not generate an utilizable result, in most cases due to the lack of integrity of the specimen.

The diagnostic spectrum of benign diseases was dominated by non-specific inflammatory reactions with the addition of consecutive tissue fibrosis and benign tumors. Lymphonodal tuberculosis or regionally predominant diseases such as schistosomiasis were rare, maybe as a result of local health campaigns and education.

In the group of benign diseases only rare cases occurred where the differential diagnosis was not completely solvable by TP. Several cases were nonetheless sent to Germany for reasons of diagnostic security. Especially the assessment of benign mesenchymal tumors (for instance the distinction between of cutaneous neurofibroma versus dermatofibroma or between non-specific inflammatory and lymphoproliferative diseases) sometimes necessitated a second opinion. The comparative data between the initial TP-diagnosis and the final diagnosis does not represent a cross section of the whole patient collective but rather provides insight into those cases which showed the highest level of diagnostic difficulty. Here in particular the interpretation 
of the definitive results in comparison to the TP-diagnoses displayed the requirement for additional reassurance as only $47 \%$ of these cases showed a complete concordance. In a further $21 \%$ mild discordances still rendered the diagnosis as useful for further therapeutic steps. Overall, a result of $12 \%$ initially false benign or false malignant diagnoses is unsatisfactory.

The group of malignant diseases was dominated by juvenile immature sarcomas and aggressive lymphomas. The rate of requisite secondary appraisal in Germany was high with $75 \%$ due to the clinical importance, the need for a high degree of diagnostic assurance and the limitations of diagnostics without additional methods. This was also demonstrated by a low number of cases which were primarily solvable with a correct diagnosis using TP of only $29 \%$ and a likewise low case number with only mild discordances $(22 \%) .15 \%$ of cases were classified as false benign. One reason for this was the substandard quality of specimen processing in the local laboratory. The same applies for the most part to $8 \%$ of cases which could not be primarily resolved via TP. An insufficient accuracy of pure TP without supplementary studies for malignant diseases, especially from the field of hematooncology, has previously been described by others. Carey et al. found a correct diagnosis in $52 \%$ of the samples ${ }^{10}$. Better results have in fact being obtained when pathologists with specialized training were deployed locally. In these cases the error rate dropped to $6.5 \%$, whereas in the project of Santiago et al. a benchmark error rate of $30 \%$ was presumed ${ }^{11}$.

One source of concern was the persistently challenging personnel situation. The training of technical assistants with no prior knowledge of histopathology is time-consuming and requires frequent phases of attendance from German volunteers, which were made possible in time blocks of up to ten weeks. Through the transfer of staff to different sectors of the public health service, work overload and alternating dedication to the project no permanent team could be established. This sometimes led to a loss of knowledge and an inconsistent quality of specimen processing. Furthermore, the local circumstances in Peramiho do not allow for the establishment of specialized stains or immunohistochemistry in time. Therefore it is of great importance that in addition to the dispatch of diagnostic cases increasing support is given to local centralized institutes, aiming to strengthen their diagnostic capacity to assume responsibility for these tasks in the future.

\section{Conclusion}

Altogether we can conclude after ten years of experience in this field, that an exclusively telepathological support of a clinic without their own local pathologists cannot meet the requirements of modern diagnostics in the special field of pediatric diseases. It can be assumed that the qualitative requirements towards histopathological diagnostics will rise in low income countries in line with the advancement of medical care. The training of local pathologists in these countries is therefore imperative. Telepathological conferences with consultant pathologists can then lead to a real improvement of the situation ${ }^{12-15}$. Until these structures are fully developed the drawbacks of static telepathology, especially in the complex field of pediatric diagnostics, must be acknowledged by treating clinicians and integrated into therapeutic decisions. Experience from daily practice shows that the direct discussion of findings between clinicians and pathologists is an essential building block in the development of a treatment concept.

In the case of new projects it will be necessary to examine the options and possibilities for further development of local laboratories and technical staff from the start. The reinforcement of expertise on-site must be given preference with the aim of establishing future self-sufficiency.

\section{Data protection and ethics}

For our retrospective analysis of completely anonymised data according to the Declaration of Helsinki, we obtained a statement of safety from the ethics committee of the University of Wuerzburg, Versbacher Straße 9, D-97078 Wuerzburg, reference number 2017060802.

\section{Patient consent}

Not required.

\section{Declaration of Conflicting Interests}

The authors declared no conflicts of interest with respect to the research, authorship and/or publication of this article.

\section{Funding}

The authors received no financial support for the research, authorship and/or publication of this article.

\section{Author's contributions}

HUV, HKMH and GS designed the study. LP, HUV, AS1 were involved in data collection, analysis and HUV, GS, HKMH, AS2 in interpretation. HUV drafted the 
manuscript. All authors critically reviewed the manuscript and approved the final version. All authors can take responsibility for the integrity or the accuracy of any part of the work.

\section{Acknowledgement}

We are deeply grateful to the technical staff at Peramiho, especially to Mr. Andrew Ponsiano and his colleagues, for their excellent work preparing the specimens for the telepathological procedure.

\section{References}

1. Sayed S, Cherniak W, Lawler M, et al. Improving pathology and laboratory medicine in low-income and middle-income countries: roadmap to solutions. Lancet 2018;391:1939-1952 PubMed

2. Brauchli K, Christen H, Haroske G, et al. Telemicroscopy by the Internet revisited. J Pathol 2002; 196(2):238-243

3. Brauchli K, Oberli H, Hurwitz N, et al. Diagnostic telepathology: long-term experience of a single institution. Virchows Arch 2004;444(5):403-409. PubMed

4. Voelker HU, Stauch G, Strehl A, et al. Diagnostic validity of static telepathology supporting hospitals without local pathologists in low-income countries. J Telemed Telecare 2018, Dec 30:1357633X18818745. doi: 10.1177/1357633X18818745. Epub ahead of print

5. Shaw EC, Hanby AM, Wheeler K, et al. Observer agreement comparing the use of virtual slides with glass slides in the pathology review component of the POSH breast cancer cohort study. J Clin Pathol 2012; 65(5):403-408

6. Wu XR, Liu HS, Shi XY, et al. Interobserver Agreement in the Diagnosis of Inflammatory Bowel Disease-Associated Neoplasia in China in Comparison to Subspecialized American Gastrointestinal Pa- thologists. Gastroenterol Res Pract 2018; 23:8715263. doi: 10.1155/2018/8715263

7. Desai S, Patil R, Kothari A et al. Static telepathology consultation service between Tata Memorial Centre, Mumbai and Nargis Dutt Memorial Charitable Hospital, Barshi, Solapur, Maharashtra: an analysis of the first 100 cases. Indian J Pathol Microbiol 2004; 47(4):480-485

8. Nalugo M, Craner DR, Schwachter M, et al. What is "telemedicine" and what does it mean for a pediatric surgeon? Eur J Pediatr Surg 2014;24(4):295-302 9. Vaught J, Abayomi A, Peakman T, et al. Critical issues in international biobanking. Clin Chem 2014; 60(11):1368-1374

10. Carey P, Fudzulani R, Scholfield D, et al. Remote and rapid pathological diagnosis in a resource challenged unit. J Clin Pathol 2014;67(6):540-543

11. Santiago TC, Jenkins JJ, Pedrosa F, et al. Improving the histopathologic diagnosis of pediatric malignancies in a low-resource setting by combining focused training and telepathology strategies. Pediatr Blood Cancer 2012;59(2):221-225

12. Ryskal O, Muschinskaja M, Göbel U, et al. Telemicroscopic conferences for children of the Perm territory with suspected or proven malignant solid tumors. Klin Padiatr 2010;222(3):199-202

13. Hartvigsen G, Johansen MA, Hasvold P, et al. Challenges in telemedicine and eHealth: lessons learned from 20 years with telemedicine in Tromsø. Stud Health Technol Inform 2007;129(1):82-86

14. Montgomery ND, Tomoka T, Krysiak R, et al. Practical Successes in Telepathology Experiences in Africa. Clin Lab Med 2018;38(1):141-150

15. Sohani AR, Sohani MA. Static digital telepathology: a model for diagnostic and educational support to pathologists in the developing world. Anal Cell Pathol (Amst) 2012;35(1):25-30 